\title{
Multi-D Upwinding and Multigridding for Steady Euler Flow Computations
}

\author{
B. Koren, P.W. Hemker \\ Center for Mathematics and Computer Science \\ P.O. Box 4079, 1009 AB Amsterdam, The Netherlands
}

\begin{abstract}
Multi-dimensional upwind discretizations for the steady Euler equations are studied, with the emphasis on both a good accuracy and a good efficiency. The discretizations consist of a one-dimensional Riemann solver with locally rotated left and right cell face states, the rotation angle depending on the local flow solution. First, on the basis of a linear, scalar model equation, a study is made of the accuracy and stability properties of these schemes. Next the extension is made to the steady Euler equations. It is shown that for Euler flows, an appropriate local rotation angle can be found by maximizing a Riemann invariant along the middle subpath of the wave path in state space. For the steady, two-dimensional Euler equations, numerical results are presented for some supersonic test cases with either oblique contact discontinuity or oblique shock wave.
\end{abstract}

Note: This work was supported by the European Space Agency (ESA), through Avions Marcel Dassault - Bréguet Aviation (AMD-BA).

\section{Introduction}

Many upwind schemes used in multi-dimensional (multi-D) flow computations are based on the application of some one-dimensional (1-D) shock capturing scheme in a grid-aligned manner. Despite the rigorous mathematics involved in these 1-D upwind schemes, in most multi-D flow computations, the underlying 1-D upwind results are just superposed without rigorous mathematical justification. Besides this inconsistency in methodology, the grid-alignment (i.e. grid-dependency) is also inconsistent with the upwind principle that discretizations should be dependent on the solution only. It seems most natural nowadays to no longer ignore the multi-D nature of a multi-D flow in the upwind scheme itself. Various grid-decoupled multi-D upwind discretizations have been published already and many more are still in development, a development which is at a rapid pace. The emphasis in most of this research clearly lies on a good accuracy.

In the present paper, for the steady Euler equations in a cell-centered finite volume context we present: multi-D upwind methods with some optimal balance between accuracy and efficiency. The steady equations are solved directly (so not through an unsteady form). For good efficiency we rely on nonlinear multigrid (multigrid-Newton) iteration. As the smoothing technique in the multigrid iteration we apply point Gauss-Seidel relaxation, using the exact derivative matrices (exact Newton). The latter requires the cell face fluxes to be continuously differentiable. If for a multi-D scheme the multigrid solver does not meet our standards we rely on defect correction iteration as the most outer iteration, just as in e.g. [1]. The multi-D upwind schemes to be considered here are very simple schemes. They use neither decoupling of the Euler equations as in [2,3], nor rotated fluxes as in [4,5]. The schemes are based on rotated left and right cell face states solely. Per cell face, just as with grid-aligned 1-D upwind schemes, only a single numerical flux is computed: the one normal to the cell face. The only difference between grid-aligned 1-D upwind schemes and the present multi-D upwind schemes is that whereas in the first schemes the left and right cell face states are computed from a solution-independent, 1-D subset of the local multi-D solution, in the present multi-D upwind schemes, these states are computed from a solution-dependent, multi- $D$ subset. The numerical flux function to be applied should allow a good resolution of both oblique shock waves and oblique contact discontinuities, which makes flux difference splitting schemes to be preferred above flux splitting schemes. Given the good experience with Osher's scheme [6] in combination with nonlinear multigrid [7], we apply this flux difference splitting scheme. 


\section{First-order accurate upwind schemes for a model equation}

For sake of simplicity, in the present paper we still restrict ourselves to first-order accurate, multi- $D$ upwind schemes. We make an analysis of such schemes for the linear, scalar, 2-D model equation

$$
a \frac{\partial u}{\partial x}+b \frac{\partial u}{\partial y}=0, \quad 0 \leq \theta \equiv \operatorname{atan}\left(\frac{b}{a}\right) \leq \frac{\pi}{2},
$$

where $\theta$ is the angle between the characteristic direction and the $x$-axis. Discretization of the model equation on a square, cell-centered finite volume grid yields

$$
a\left(u_{i+\frac{1}{2}, j}-u_{i-\frac{1}{2}, j}\right)+b\left(u_{i, j+\frac{1}{2}}-u_{i, j-\frac{1}{2}}\right)=0
$$

where the half-integer indices refer to the cell faces between the (full-integer indexed) cell centers. Taking into account the positive signs of $a$ and $b$, various first-order upwind choices

$$
\left(\begin{array}{l}
u_{i+\frac{1}{2}, j} \\
u_{i, j+\frac{1}{2}}
\end{array}\right)=\left(\begin{array}{c}
L_{h}\left(u_{i, j}, u_{i, j-1}, u_{i+1, j-1}\right) \\
L_{v}\left(u_{i, j}, u_{i-1, j}, u_{i-1, j+1}\right)
\end{array}\right), \quad 0 \leq \theta \leq \frac{\pi}{2},
$$

can be made, which (with similar choices for $u_{i-\frac{1}{2}, j}$ and $u_{i, j-\frac{1}{2}}$ ) lead to the general 6-point compact stencil

$$
\left[\begin{array}{ccc}
-\alpha_{i-1, j+1} & \cdot & \cdot \\
-\alpha_{i-1, j} & \alpha_{i, j} & \cdot \\
-\alpha_{i-1, j-1} & -\alpha_{i, j-1} & -\alpha_{i+1, j-1}
\end{array}\right], \quad 0 \leq \theta \leq \frac{\pi}{2} .
$$

A general stability requirement imposed to these schemes is that they satisfy the positive coefficients rule [8]. (Schemes satisfying this rule do not allow unstable, oscillatory solutions.)

\section{Definition 1}

Consider the general stencil (4). In this paper, schemes satisfying the positive coefficients rule

$$
\alpha_{i, j} \geq 0, \quad \alpha_{i \pm m, j \pm n} \geq 0, \quad \forall m, n \in \mathbb{N},
$$

are called positive.

Applying truncated Taylor series expansions to the discrete equation corresponding to (4) and transforming to characteristic coordinates, one derives a modified equation of the general form

$$
\frac{\partial u}{\partial s}-\frac{h}{2}\left[\mu_{s s} \frac{\partial^{2} u}{\partial s^{2}}+\mu_{s n} \frac{\partial^{2} u}{\partial s \partial n}+\mu_{n n} \frac{\partial^{2} u}{\partial n^{2}}\right]=\mathrm{O}\left(h^{2}\right) .
$$

In the present paper, we prefer multi-D upwind schemes with both $\mu_{s n}=0$ and $\mu_{n n}=0$; discretizations which guarantee a low crosswind diffusion for $\frac{\partial u}{\partial s}=r(s, n)$, with $r(s, n)$ arbitrary.

Definition 2

Consider the general modified equation (6). In this paper, $\left(\mu_{s n}, \mu_{n n}\right)^{T}$ is called the crosswind diffusion and schemes for which $\left(\mu_{s n}, \mu_{n n}\right)^{T}=0$ are called zero-crosswind diffusion schemes.

\subsection{The standard, grid-aligned scheme}

With the standard, first-order accurate, grid-aligned 1-D upwind scheme, given the positive signs of $a$ and $b$, for the cell face states occurring in (2) one takes

$$
\left(\begin{array}{l}
u_{i+\frac{1}{2}, j} \\
u_{i, j+\frac{1}{2}}
\end{array}\right)=\left(\begin{array}{l}
u_{i, j} \\
u_{i, j}
\end{array}\right), \quad 0 \leq \theta \leq \frac{\pi}{2},
$$

with similar choices for $u_{i-\frac{1}{2}, j}$ and $u_{i, j-\frac{1}{2}}$. The scheme is positive, which clearly appears from its stencil

$$
\left[\begin{array}{ccc}
\cdot & \cdot & \cdot \\
-a & a+b & \cdot \\
\cdot & -b & \cdot
\end{array}\right], \quad 0 \leq \theta \leq \frac{\pi}{2} .
$$

Further it is continuously differentiable, which allows the application of Newton iteration. However, applying the modified equation approach, one finds the poor accuracy properties given in Fig. 1a. It 
appears that zero-crosswind diffusion occurs only in case of $\theta=0$ or $\theta=\frac{\pi}{2}$, i.e. in case of grid-alignment of the characteristic direction. The weak spot of scheme (7) is of course that it only uses two off-diagonal points. In constructing multi-D upwind schemes it is self-evident to add at least $u_{i-1, j-1}$ to the scheme. In the following we give two first-order accurate, multi-D upwind schemes in which this has been done.

\subsection{A positive, continuously differentiable scheme}

The present scheme is derived in [9]. (In another way and in another context, it is also derived in [10].) For the cell face states it takes:

$$
\left(\begin{array}{c}
u_{i+\frac{1}{2}, j} \\
u_{i, j+\frac{1}{2}}
\end{array}\right)=\frac{1}{a+b}\left(\begin{array}{c}
\left(a+\frac{1}{2} b\right) u_{i, j}+\frac{1}{2} b u_{i, j-1} \\
\left(b+\frac{1}{2} a\right) u_{i, j}+\frac{1}{2} a u_{i-1, j}
\end{array}\right), \quad 0 \leq \theta \leq \frac{\pi}{2}
$$

leading to the stencil

$$
\frac{1}{a+b}\left[\begin{array}{ccc}
\cdot & \cdot & \cdot \\
-a^{2} & a^{2}+a b+b^{2} & \cdot \\
-a b & -b^{2} & \cdot
\end{array}\right], \quad 0 \leq \theta \leq \frac{\pi}{2},
$$

and the diffusion coefficients given in Fig. 1b. The scheme has been constructed such that $\mu_{s n}=0$ over the complete range of $\theta$ considered. Notice that compared to scheme (7), also $\mu_{n n}$ is smaller. Moreover, in [9] it is shown that this scheme still allows a more efficient smoothing of point Gauss-Seidel relaxation than scheme (7). All this makes that it is a more appropriate candidate for our multigrid purposes than scheme (7).

\subsection{A zero-crosswind diffusion scheme}

Following the modified equation approach, in [9] the derivation is also given of the 6-point compact scheme

$$
\begin{gathered}
\left(\begin{array}{l}
u_{i+\frac{1}{2}, j} \\
u_{i, j+\frac{1}{2}}
\end{array}\right)=\left(\begin{array}{c}
u_{i, j} \\
\frac{1}{2}\left(1+\frac{b}{a}\right) u_{i-1, j}+\frac{1}{2}\left(1-\frac{b}{a}\right) u_{i-1, j+1}
\end{array}\right), \quad 0 \leq \theta \leq \frac{\pi}{4}, \\
\left(\begin{array}{l}
u_{i+\frac{1}{2}, j} \\
u_{i, j+\frac{1}{2}}
\end{array}\right)=\left(\begin{array}{c}
\frac{1}{2}\left(1+\frac{a}{b}\right) u_{i, j-1}+\frac{1}{2}\left(1-\frac{a}{b}\right) u_{i+1, j-1} \\
u_{i, j}
\end{array}\right), \quad \frac{\pi}{4} \leq \theta \leq \frac{\pi}{2},
\end{gathered}
$$

leading to the stencils

$$
\begin{gathered}
{\left[\begin{array}{ccc}
\frac{1}{2} b\left(1-\frac{b}{a}\right) & \cdot & \cdot \\
b\left(\frac{b}{a}-\frac{a}{b}\right) & a & \cdot \\
-\frac{1}{2} b\left(1+\frac{b}{a}\right) & \cdot & \cdot
\end{array}\right], \quad 0 \leq \theta \leq \frac{\pi}{4},} \\
\left.\cdot \begin{array}{ccc}
\cdot & \cdot & \cdot \\
-\frac{1}{2} a\left(1+\frac{a}{b}\right) & a\left(\frac{a}{b}-\frac{b}{a}\right) & \frac{1}{2} a\left(1-\frac{a}{b}\right)
\end{array}\right], \quad \frac{\pi}{4} \leq \theta \leq \frac{\pi}{2},
\end{gathered}
$$

and the diffusion coefficients given in Fig. 1c. This scheme has been constructed such that it has $\left(\mu_{s n}, \mu_{n n}\right)^{T}=0$ over the complete range of $\theta$ considered. Concerning the solution of the corresponding discretized equations, due their non-positivity, no such efficient smoother exists as for the equations belonging to scheme (9). To solve the present equations we rely on defect correction iteration with as 'working horse' scheme in the inner multigrid iteration: scheme (9). In [9] it is shown that this is a satisfactory approach.

\section{The foregoing schemes for the Euler equations}

We proceed by extending the foregoing model schemes to the steady, 2-D Euler equations. The extension is straightforward. Because in the model equation the characteristic information is coming from the left, for the Eulerian numerical flux function the components of the left cell face states are computed in the same way as the cell face states for the model equation. For the right cell face states, we simply take the point symmetric counterpart of the left states. In this way, in case of all characteristic information coming from the right (supersonic flow from the right), one also has the proper discretization. Also notice that in this way an $n$-point scheme for the model equation becomes a $(2 \times n-1)$-point scheme for the Euler equations. In mathematical formulae, the procedure reads as follows. Consider as general relation for the left cell face states to be substituted into the numerical flux function:

$$
\left(\begin{array}{c}
q_{i+\frac{1}{2}, j} \\
q_{i, j+\frac{1}{2}}
\end{array}\right)^{l}=\left(\begin{array}{c}
L_{h}\left(q_{i, j}, q_{i, j-1}, q_{i+1, j-1}\right) \\
L_{v}\left(q_{i, j}, q_{i-1, j}, q_{i-1, j+1}\right)
\end{array}\right), \quad 0 \leq \theta \leq \frac{\pi}{2},
$$


then the right cell face states simply obey:

$$
\left(\begin{array}{c}
q_{i+\frac{1}{2}, j} \\
q_{i, j+\frac{1}{2}}
\end{array}\right)^{r}=\left(\begin{array}{c}
L_{h}\left(q_{i+1, j}, q_{i+1, j+1}, q_{i, j+1}\right) \\
L_{v}\left(q_{i, j+1}, q_{i+1, j+1}, q_{i+1, j}\right)
\end{array}\right), \quad 0 \leq \theta \leq \frac{\pi}{2} .
$$

3.1. The standard, grid-aligned scheme

For sake of completeness we also make the extension for scheme (7). For this scheme we get the known result

$$
\begin{gathered}
\left(\begin{array}{l}
q_{i+\frac{1}{2}, j} \\
q_{i, j+\frac{1}{2}}
\end{array}\right)^{l}=\left(\begin{array}{l}
q_{i, j} \\
q_{i, j}
\end{array}\right), \quad 0 \leq \theta \leq \frac{\pi}{2}, \\
\left(\begin{array}{l}
q_{i+\frac{1}{2}, j} \\
q_{i, j+\frac{1}{2}}
\end{array}\right)^{r}=\left(\begin{array}{l}
q_{i+1, j} \\
q_{i, j+1}
\end{array}\right), \quad 0 \leq \theta \leq \frac{\pi}{2} .
\end{gathered}
$$

3.2. The positive, continuously differentiable scheme Positive, continuously differentiable scheme (9) as derived for model equation (1), is applied as

$$
\begin{gathered}
\left(\begin{array}{c}
q_{i+\frac{1}{2}, j} \\
q_{i, j+\frac{1}{2}}
\end{array}\right)^{l}=\frac{1}{1+\tan \theta}\left(\begin{array}{c}
\left(1+\frac{1}{2} \tan \theta\right) q_{i, j}+\frac{1}{2} \tan \theta q_{i, j-1} \\
\left(\frac{1}{2}+\tan \theta\right) q_{i, j}+\frac{1}{2} q_{i-1, j}
\end{array}\right), \quad 0 \leq \theta \leq \frac{\pi}{2}, \\
\left(\begin{array}{c}
q_{i+\frac{1}{2}, j} \\
q_{i, j+\frac{1}{2}}
\end{array}\right)^{r}=\frac{1}{1+\tan \theta}\left(\begin{array}{c}
\left(1+\frac{1}{2} \tan \theta\right) q_{i+1, j}+\frac{1}{2} \tan \theta q_{i+1, j+1} \\
\left(\frac{1}{2}+\tan \theta\right) q_{i, j+1}+\frac{1}{2} q_{i+1, j+1}
\end{array}\right), \quad 0 \leq \theta \leq \frac{\pi}{2} .
\end{gathered}
$$

3.3. The zero-crosswind diffusion scheme

Similarly, zero-crosswind diffusion scheme (11) is applied as

$$
\begin{gathered}
\left(\begin{array}{c}
q_{i+\frac{1}{2}, j} \\
q_{i, j+\frac{1}{2}}
\end{array}\right)^{l}=\left(\begin{array}{c}
q_{i, j} \\
\frac{1}{2}(1+\tan \theta) q_{i-1, j}+\frac{1}{2}(1-\tan \theta) q_{i-1, j+1}
\end{array}\right), \quad 0 \leq \theta \leq \frac{\pi}{4}, \\
\left(\begin{array}{c}
q_{i+\frac{1}{2}, j} \\
q_{i, j+\frac{1}{2}}
\end{array}\right)^{l}=\left(\begin{array}{c}
\frac{1}{2}\left(1+\frac{1}{\tan \theta}\right) q_{i, j-1}+\frac{1}{2}\left(1-\frac{1}{\tan \theta}\right) q_{i+1, j-1} \\
q_{i, j}
\end{array}\right), \quad \frac{\pi}{4} \leq \theta \leq \frac{\pi}{2},
\end{gathered}
$$

and

$$
\begin{aligned}
& \left(\begin{array}{c}
q_{i+\frac{1}{2}, j} \\
q_{i, j+\frac{1}{2}}
\end{array}\right)^{r}=\left(\begin{array}{c}
q_{i+1, j} \\
\frac{1}{2}(1+\tan \theta) q_{i+1, j+1}+\frac{1}{2}(1-\tan \theta) q_{i+1, j}
\end{array}\right), \quad 0 \leq \theta \leq \frac{\pi}{4}, \\
& \left(\begin{array}{c}
q_{i+\frac{1}{2}, j} \\
q_{i, j+\frac{1}{2}}
\end{array}\right)^{r}=\left(\begin{array}{c}
\frac{1}{2}\left(1+\frac{1}{\tan \theta}\right) q_{i+1, j+1}+\frac{1}{2}\left(1-\frac{1}{\tan \theta}\right) q_{i, j+1} \\
q_{i, j+1}
\end{array}\right), \quad \frac{\pi}{4} \leq \theta \leq \frac{\pi}{2} .
\end{aligned}
$$

\section{Rotation angle for the Euler equations}

Per cell face we have to select a single rotation angle from the local, multi-D solution. Here we present a technique which looks at all cell faces at either the local flow angle or a local shock wave angle. The technique considers a wave path in state space. As the wave path we consider the one of the P-variant of Osher's scheme [7]. For the steady 2-D Euler equations and a perfect gas, with $c \equiv \sqrt{\gamma_{\rho}^{p}}$ and $z \equiv \ln \left(\frac{p}{\rho^{\gamma}}\right)$, the P-variant's wave path is shown in Fig. 2. For the determination of the angle $\theta$, the wave path states $q_{0} \equiv\left(u_{0}, v_{0}, c_{0}, z_{0}\right)^{T}$ and $q_{1} \equiv\left(u_{1}, v_{1}, c_{1}, z_{1}\right)^{T}$ are taken dependent on $\theta$ as

$$
q_{0}(\theta)=T(\theta) q^{l}, \quad q_{1}(\theta)=T(\theta) q^{\top},
$$

with

$$
T(\theta)=\left(\begin{array}{cccc}
\cos \theta & \sin \theta & 0 & 0 \\
-\sin \theta & \cos \theta & 0 & 0 \\
0 & 0 & 1 & 0 \\
0 & 0 & 0 & 1
\end{array}\right)
$$


and with the states $q^{l}$ and $q^{r}$ given. At the cell faces $i+\frac{1}{2}, j$ and $i, j+\frac{1}{2}$, for $q^{l}$ and $q^{r}$ we take e.g.

$$
\begin{gathered}
\left(\begin{array}{l}
q_{i+\frac{1}{2}, j} \\
q_{i, j+\frac{1}{2}}
\end{array}\right)^{l}=\left(\begin{array}{l}
q_{i, j} \\
q_{i, j}
\end{array}\right), \\
\left(\begin{array}{c}
q_{i+\frac{1}{2}, j} \\
q_{i, j+\frac{1}{2}}
\end{array}\right)^{r}=\left(\begin{array}{l}
q_{i+1, j} \\
q_{i, j+1}
\end{array}\right) .
\end{gathered}
$$

Then, a suitable rotation angle can be found by maximizing one of the two Riemann invariants along the middle subpath of the P-variant's wave path in state space; i.e. either the invariant velocity component

$$
u_{\frac{1}{2}}(\theta)=\frac{1}{1+\alpha}\left[\left(u_{1}(\theta)-\frac{2}{\gamma-1} c_{1}\right)+\alpha\left(u_{0}(\theta)+\frac{2}{\gamma-1} c_{0}\right)\right]
$$

or the invariant pressure

$$
p_{\frac{1}{2}}(\theta)=\left[\frac{\gamma-1}{2(1+\alpha) \sqrt{\gamma e^{\frac{x_{0}}{\gamma}}}}\left(u_{0}(\theta)-u_{1}(\theta)+\frac{2}{\gamma-1}\left(c_{0}+c_{1}\right)\right)\right]^{\frac{2 \gamma}{\gamma-1}},
$$

where in both: $\alpha \equiv e^{\frac{x_{1}-z_{0}}{\gamma_{\gamma}}}$.

\section{Theorem 1}

The orientation of a contact discontinuity follows from the maximization of the velocity component $u_{\frac{1}{2}}(\theta)$ along the $P$-variant's wave path in state space.

\section{Proof}

From (23a), it follows with (21a)-(21b):

$$
\frac{d u_{\frac{1}{2}}}{d \theta}=\frac{1}{1+\alpha}\left[-\sin \theta u^{r}+\cos \theta v^{r}+\alpha\left(-\sin \theta u^{l}+\cos \theta v^{l}\right)\right],
$$

from which it is found that $u_{\frac{1}{2}}$ is maximal for

$$
\tan \theta=\frac{\alpha v^{l}+v^{r}}{\alpha u^{l}+u^{r}}
$$

which is a biased relation for the orientation of a contact discontinuity. $\square$

\section{Remark 1}

For the isentropic case, $\alpha=1,(25)$ simplifies to the known centered relation

$$
\tan \theta=\frac{v^{l}+v^{r}}{u^{l}+u^{r}}
$$

Remark 2

The physical meaning of $(25)$ for $\alpha \neq 1$ can be explained for the isobaric case $p^{l}=p^{r}$. For this case, (25) simplifies to the (still biased) relation

$$
\tan \theta=\frac{\sqrt{\rho^{l}} v^{l}+\sqrt{\rho^{r}} v^{r}}{\sqrt{\rho^{l}} u^{l}+\sqrt{\rho^{r}} u^{r}} .
$$

From (27) it follows that of the two states $q^{l}$ and $q^{r}$, the state with the higher density has a stronger weight in the determination of the angle $\theta$. To our opinion this is physically more proper than the absence of any such weight in the commonly used centered relation (26).

Theorem 2

The orientation of the normal at a shock wave follows from the maximization of the pressure $p_{\frac{1}{2}}(\theta)$ along the $P$-variant's wave path in state space. 
Proof

From (23b), it follows with (21a)-(21b):

$$
\frac{d p_{\frac{1}{2}}}{d \theta}=\frac{1}{1+\alpha} \sqrt{\frac{\gamma p_{\frac{1}{2}}^{\frac{\gamma+1}{\gamma}}}{e^{\frac{\frac{2}{\gamma}}{\gamma}}}}\left(-\sin \theta\left(u^{l}-u^{r}\right)+\cos \theta\left(v^{l}-v^{r}\right)\right),
$$

from which it is found that $p_{\frac{1}{2}}$ is maximal for

$$
\tan \theta_{n}=\frac{v^{l}-v^{r}}{u^{l}-u^{r}}
$$

This is a relation for the orientation of the normal at a shock wave, because - given the $\theta$-independence of $p_{0}$ and $p_{1}$ - the maximization of $p_{\frac{1}{2}}$ is identical to the maximization of either $p_{\frac{1}{2}}-p_{0}$ or $p_{\frac{1}{2}}-p_{1}$; the underlying, natural quantities for finding the orientation of the normal at a shock wave.

\section{Corollary}

The shock wave angle itself, i.e. the rotation angle $\theta$, satisfies

$$
\tan \theta=\frac{u^{l}-u^{r}}{v^{r}-v^{l}}
$$

Remark 3

In contrast with (25), the result (30) is known. It directly follows from the jump relation which states that the tangential velocity components at the up- and downstream side of a shock wave are equal.

\section{Remark 4}

As opposed to (25), (30) contains differences, which makes it sensitive to noise and non-uniqueness. In principle, as a remedy against this, a blended formula like that proposed in [5], can also be constructed on the basis of (25) and (30).

Remark 5

Notice that by taking the shock wave angle as rotation angle, the upwinding is not done normal to the shock wave, cf. e.g. Davis [4], but - instead - along the shock wave; i.e. along the (merged) characteristics.

\section{Numerical results}

For the steady, 2-D Euler equations and a perfect gas with $\gamma=1.4$, numerical experiments are performed for some supersonic, unit square flows with either oblique contact discontinuity or oblique shock wave. First, flows with contact discontinuity are considered for the flow angles $\theta=0.1 \pi, 0.2 \pi, 0.3 \pi$ and $0.4 \pi$ (Fig. 3a). Next, flows with shock wave are considered for the shock wave angles $\theta=\frac{\pi}{4}$ and $\theta=\frac{\pi}{8}$ (Fig. $3 \mathrm{~b})$. All these flows are computed on a uniform $32 \times 32$-grid. In all cases - for simplicity - at each of the four boundaries, the exact solution is imposed (overspecification). Further, in all cases, standard nonlinear multigrid method (FAS) is applied; with a $2 \times 2$-grid as the coarsest grid, with V-cycles and with a single pre- and post-relaxation sweep per level. We remark that with scheme (17)-(18) to be locally linearized in the inner multigrid iteration, one has $4 \times 4$ derivative matrices containing contributions which originate from the solution-dependent rotation angle. In all cases we take as the initial solution: the solution with $q=q^{L}$ (the exact $q^{L}$ 's from Figs. $3 \mathrm{a}$ and $3 \mathrm{~b}$ ) uniformly constant over the complete domain.

\subsection{Flows with contact discontinuity}

First, in Fig. 4, reference results are given for the present four test cases; results obtained by the firstorder, grid-aligned 1-D upwind scheme (15)-(16). In Fig. 4a, we plotted on top of each other: the enthalpy $\left(e+\frac{p}{\rho}\right)$ distributions for $\theta=0.1 \pi, 0.2 \pi, 0.3 \pi$ and $0.4 \pi$. The iso-enthalpy values considered in these and all following enthalpy distributions are: $1.1,1.2,1.3, \ldots, 1.9$. Because of the severe smearing of the scheme, hardly any distinction can be made between the four solutions. (Notice that the layers along $x=1$ and $y=1$ in Fig. $4 \mathrm{a}$, and also in the following enthalpy graphs, are only due to the overspecification.) The convergence histories belonging to the standard, grid-aligned 1-D upwind scheme, are given in Fig. 4b.

In Fig. 5, results are given as obtained by multi-D scheme (17)-(18), with as the rotation angle: the local flow angle according to (25). Though more accurate than the grid-aligned reference distributions in 
Fig. 4a, the present enthalpy distributions (Fig. 5a) are still insufficiently accurate. Though not as very fast as the reference convergence in Fig. $4 \mathrm{~b}$, the present scheme's multigrid convergence (Fig. $5 \mathrm{~b}$ ) is still very good.

In Fig. 6a we give the enthalpy distributions for zero-crosswind diffusion scheme (19)-(20), as obtained after 10 defect correction cycles (with a single nonlinear multigrid cycle per defect correction cycle), and with also (25) for the rotation angle considered at each cell face. All these four enthalpy distributions appear to be almost free of crosswind diffusion. Although in principle the non-positivity of the scheme allows solutions with spurious oscillations, the distributions in Fig. 6a are still monotone. For comparison, in Fig. $6 \mathrm{~b}$ we still give the distributions obtained by a higher-order accurate, grid-aligned 1-D upwind scheme: the $\kappa=\frac{1}{3}$-scheme [11]. All four enthalpy distributions of first-order accurate scheme (19)-(20) appear to be even less diffused than those of the higher-order accurate $\kappa=\frac{1}{3}$-scheme !

\subsection{Flows with shock wave}

Reference results obtained by the first-order, grid-aligned 1-D upwind scheme (15)-(16) (Mach number distributions) are given in Fig. 7a. Here solutions are also plotted on top of each other. The isoMach number values shown are: (i) $1.50,1.55,1.60, \ldots, 1.95$ for the case with $\theta=\frac{\pi}{4}, M^{L}=2$, and (ii) $3.30,3.35,3.40, \ldots, 3.95$ for the case with $\theta=\frac{\pi}{8}, M^{L}=4$. (Similar to the flows with contact discontinuity, the layers along $x=1$ and $y=1$ are caused by the overspecification.) In Fig. 7b we give the Mach number distributions as obtained after two defect correction cycles with zero-crosswind diffusion scheme (19)-(20). The rotation angle considered here is the shock wave angle according to (30). After two defect correction cycles, the solution seems to be free of crosswind diffusion, but for $\theta=\frac{\pi}{4}$ it has become non-monotone. Construction of a compact multi-D limiter might be useful. For further comparisons, in Fig. 7c we still give the distributions obtained by the limited $\kappa=\frac{1}{3}$-scheme from [12]. The monotone solution as obtained for $\theta=\frac{\pi}{8}$ by the first-order accurate zero-crosswind diffusion scheme appears to be less diffused than that of the higher-order accurate $\kappa=\frac{1}{3}$-scheme.

\section{Conclusions}

Multi-D upwinding through a 1-D Riemann solver with a local, solution-dependent rotation of the left and right Riemann states, allows to keep the number of flux computations per cell face equal to one only. Good efficiency is further guaranteed through nonlinear multigrid iteration and defect correction iteration. The accuracy and efficiency of the multi-D results are promising. For flows with contact discontinuities, the performance of nonlinear multigrid with point Gauss-Seidel relaxation is (still) good when one applies the positive, continuously differentiable, 7-point compact scheme. Also for flows with contact discontinuities, the solutions obtained by the zero-crosswind diffusion, 9-point compact scheme appear to be nearly free of any crosswind diffusion. Moreover, their computation by means of defect correction iteration (with the positive, continuously differentiable, 7-point compact scheme as the approximate scheme) is efficient. The zero-crosswind diffusion scheme seems to be well-suited for an accurate and efficient computation of e.g. vortex flows

The numerical techniques presented in this paper do not require any tuning of parameters. Further, they can be carried over to $3-\mathrm{D}$ and extended to non-Cartesian grids.

\section{References}

1. Hemker, P.W.: Defect correction and higher order schemes for the multi grid solution of the steady Euler equations, Lecture Notes in Mathematics, 1228 (Springer, Berlin, 1986) pp. 149-165.

2. Hirsch, Ch., LACOR, C., DeConinck, H.: Convection algorithms based on a diagonalization procedure for the multidimensional Euler equations, AIAA paper 87-1163 (1987).

3. ROE, P.L.: Discrete models for the numerical analysis of time-dependent multidimensional gas dynamics, J. Comput. Phys., 63 (1986) pp. 458-476.

4. Davis, S.F.: A rotationally biased upwind difference scheme for the Euler equations, J. Comput. Phys., 56 (1984) pp. 65-92.

5. Levy, D.W., Powell, K.G., Van Leer, B.: An implementation of a grid-independent upwind scheme for the Euler equations, AIAA paper 89-1931 (1989). 
6. Osher, S., SOLOmon, F.: Upwind difference schemes for hyperbolic systems of conservation laws, Math. Comput., 38 (1982) pp. 339-374.

7. Hemker, P.W., SPEkreisse, S.P.: Multiple grid and Osher's scheme for the efficient solution of the steady Euler equations, Appl. Numer. Math., 2 (1986) pp. 475-493.

8. Patankar, S.V.: Numerical Heat Transfer and Fluid Flow (Hemisphere, New York, 1980).

9. KOREN, B.: Low-diffusion rotated upwind schemes, multigrid and defect correction for steady, multi-dimensional Euler flows, International Series of Numerical Mathematics, 98 (Birkhäuser, Basel, 1991) pp. 265-276.

10. LaYTON, W.: On the principal axes of diffusion in difference schemes for $2 \mathrm{D}$ transport problems, J. Comput. Phys., 90 (1990) pp. 336-347.

11. VAN LEER, B.: Upwind-difference methods for aerodynamic problems governed by the Euler equations, Lectures in Applied Mathematics, 22 (Amer. Math. Soc., Providence, RI, 1985) pp. 327-336.

12. Koren, B.: Upwind discretization of the steady Navier-Stokes equations, Int. J. Numer. Meth. Fluids, 11 (1990) pp. 99-117.

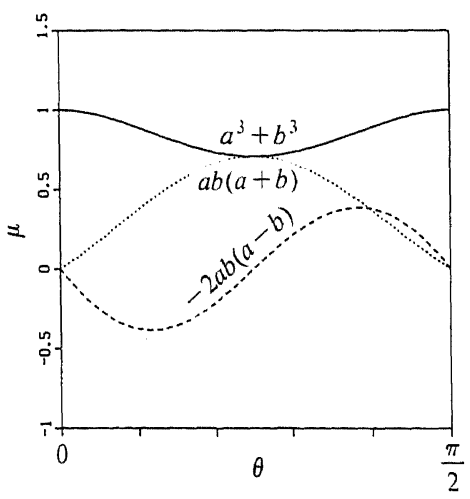

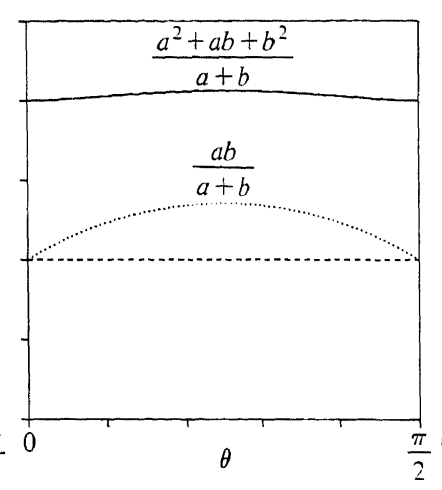

b. Positive, continuously differentiable scheme (9).

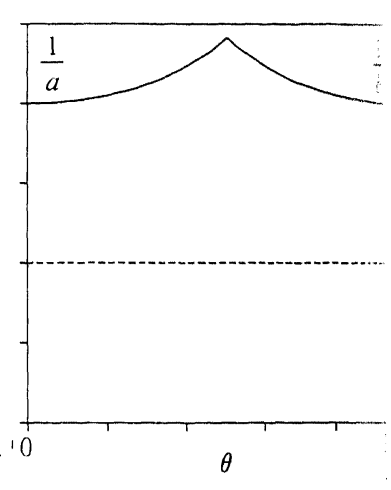

c. Zero-crosswind diffusion scheme (11).

Fig. 1. Diffusion coefficients modified equations

$$
\left(\mu_{s s}:-, \mu_{s n}:-\cdots-\cdots, \mu_{n n}: \ldots \ldots . .\right) \text {. }
$$

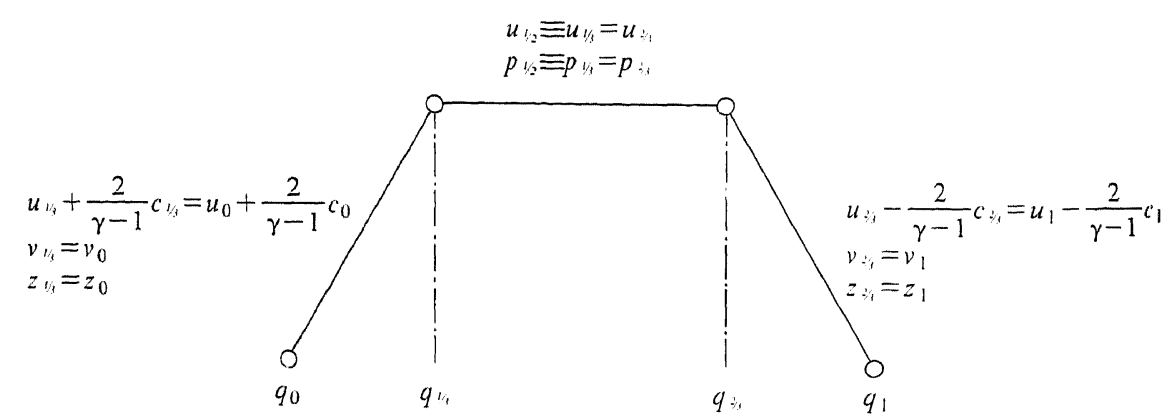

Fig. 2. Wave path in state space according to P-variant Osher scheme (perfect gas). 


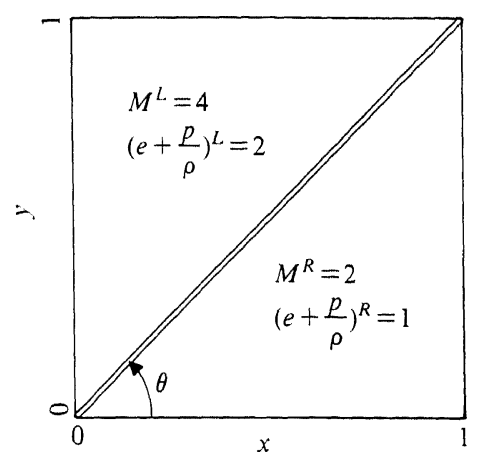

a. Oblique contact discontinuity $(\theta=0.1 \pi, 0.2 \pi, 0.3 \pi, 0.4 \pi, p=1)$.

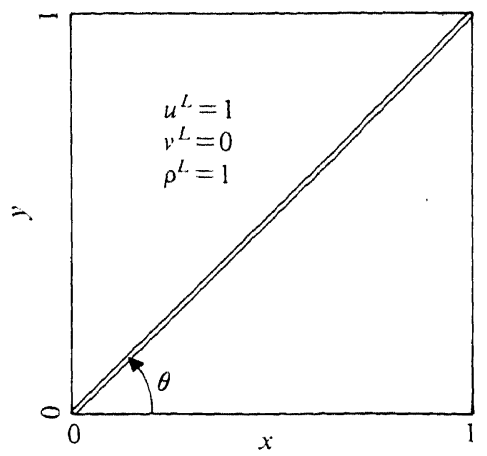

b. Oblique shock wave $\left(\theta=\frac{\pi}{4}, M^{L}=2\right.$ and $\left.\theta=\frac{\pi}{8}, M^{L}=4\right)$.

Fig. 3. Test cases to be considered on unit square.

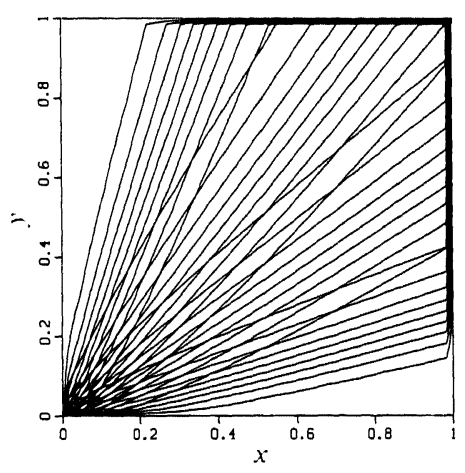

a. Enthalpy distributions.

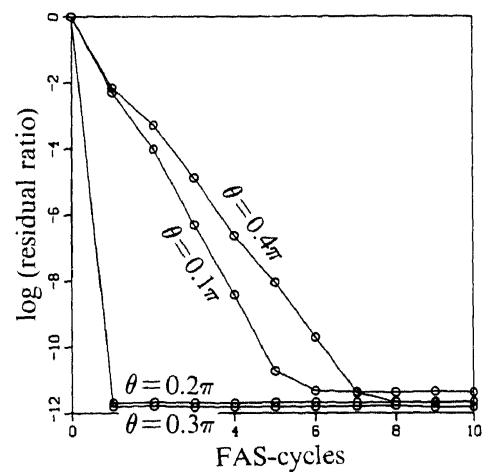

b. Multigrid convergence histories.

Fig. 4. Results standard, grid-aligned scheme (15)-(16), flows with contact discontinuity.

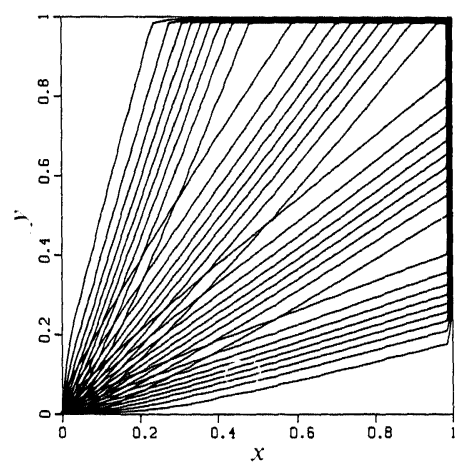

a. Enthalpy distributions.

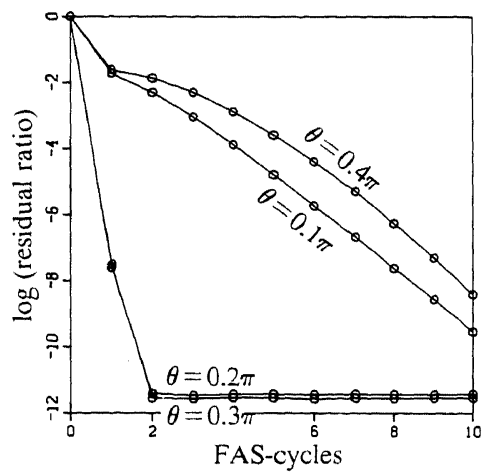

b. Multigrid convergence histories.

Fig. 5. Results positive, continuously differentiable scheme (17)-(18), flows with contact discontinuity. 


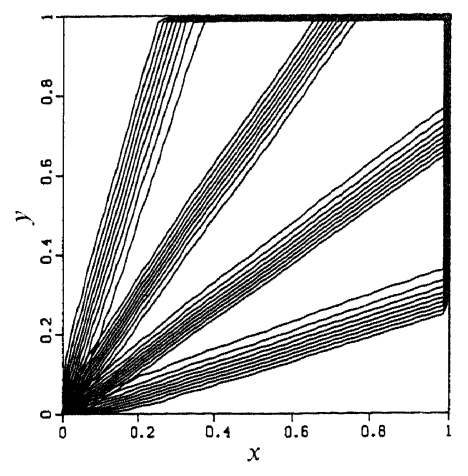

a. Zero-crosswind diffusion scheme (19)-(20).

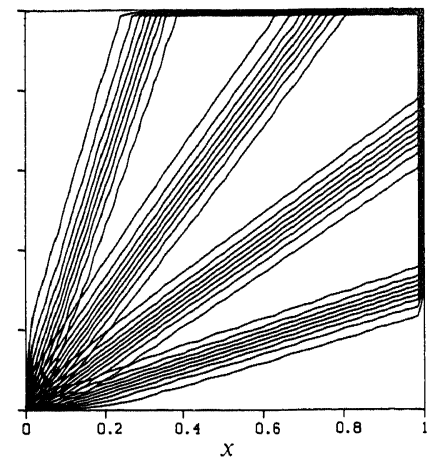

b. Non-limited $\kappa=1 / 3$-scheme [11].

Fig. 6. Enthalpy distributions, flows with contact discontinuity.

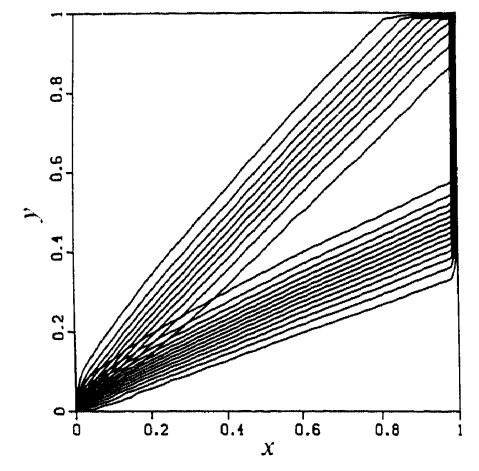

a. Standard, grid-aligned upwind scheme (15)-(16).

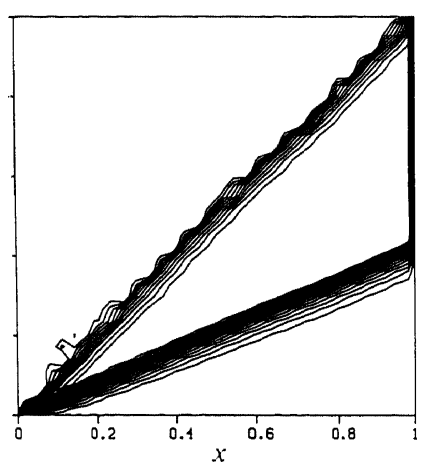

b. Zero-crosswind diffusion scheme (19)-(20).

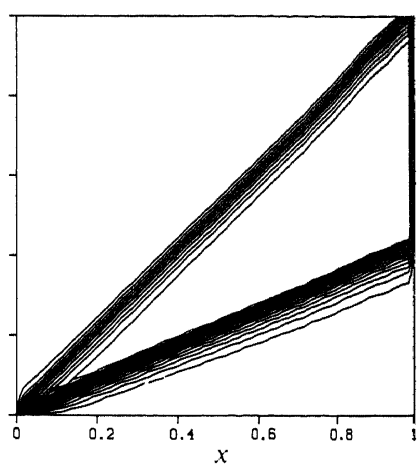

c. Limited $\kappa=1 / 3$-scheme [12].

Fig. 7. Mach number distributions, flows with shock wave. 